\title{
ABORDAGEM DA SAÚdE DA POPULAÇÃO NEGRA NOS CURSOS DA ÁREA DE SAÚDE
}

\author{
ENFOQUE DE LA SALUD DE LA POBLACIÓN NEGRA EN LOS CURSOS DEL ÁREA \\ DE SALUD
}

\author{
ADDRESSING HEALTH OF THE BLACK POPULATION IN THE HEALTH \\ EDUCATION
}

\author{
Ana Carolina Lino SILVERIO ${ }^{1}$ \\ Nicole Geovana DIAS ${ }^{2}$
}

RESUMO: O estudo da Saúde da População Negra é importante para identificar e solucionar as questões que afetam a saúde dessa população, dentre estas, o racismo. A Saúde da População Negra não é uma área de conhecimento priorizada nos cursos da área da Saúde, apesar de essencial para a formação dos profissionais de saúde e para a própria população negra. Essas insuficiências podem indicar o não reconhecimento das necessidades desta população impactando nos índices da desigualdade. Esse estudo tem como objetivo apurar, nos cursos de Saúde da Universidade Federal de Uberlândia, a existência de conteúdo sobre Saúde da População Negra, tais como disciplinas, ementas e planos de ensino. Como resultados obtivemos poucos achados de abordagem da Saúde da População Negra. Contudo, apresentamos potencialidades de onde esse importante tema pode ser abordado nas disciplinas dos cursos da área de saúde.

PALAVRAS-CHAVE: Políticas públicas de saúde. Saúde das minorias étnicas. Educação em saúde.

RESUMEM : El estudio de la Salud de la Población Negra es importante para identificar y solucionar las cuestiones que afectan la salud de esa población, entre ellas, el racismo. La Salud de la Población Negra no es un área de conocimiento priorizada en los cursos del área de la Salud, a pesar de ser esencial para la formación de los profesionales de salud y para la propia población negra. Estas deficiencias pueden indicar el no reconocimiento de las necesidades de esta población impactando en los índices de desigualdad. Este estudio tiene como objetivo apurar, en los cursos de Salud de la Universidad Federal de Uberlandia, la existencia de contenido sobre Salud de la Población Negra, tales como disciplinas, menús y planes de enseñanza. Como resultados obtuvimos pocos hallazgos de abordaje de la Salud de la Población Negra. Sin embargo, presentamos posibles potencialidades de donde ese importante tema puede ser abordado en las disciplinas de los cursos del área de salud.

${ }^{1}$ Universidade Federal de Uberlândia (UFU), Uberlândia - MG - Brasil. Fisioterapeuta formada pela Universidade Federal de Uberlândia (UFU). ORCID: <http://orcid.org/0000-0002-7940-3114>. E-mail: anacarolinalino.fisioterapia@gmail.com

${ }^{2}$ Universidade Federal de Uberlândia (UFU), Uberlândia - MG - Brasil. Docente do Departamento de Saúde Coletiva. ORCID: <http://orcid.org/0000-0002-8095-6664>.E-mail: nicole.geovana@ufu.br 
PALABRAS CLAVE: Políticas públicas de salud. Salud de las minorías étnicas. Educación en salud.

ABSTRACT: The study of the Health of the Black Population is important to identify and solve the issues that affect the health of this population, among them, racism. The Health of the Black Population is not a prioritized knowledge area in the courses of the Health area, although essential for the training of health professionals and for the black population itself. These shortcomings may indicate the non-recognition of the needs of this population impacting inequality indices. This study aims to find out, in the Health courses of the Federal University of Uberlândia, the existence of content on Health of the Black Population, such as disciplines, menus and teaching plans. As results we obtained few findings of approach of the Health of the Black Population. However, we present possible potentialities from which this important theme can be approached in the disciplines of the health courses.

KEYWORDS: Public health policy. Health of ethnic minorities. Health education.

\section{Introdução}

O estudo da Saúde da População Negra é de grande importância para identificar e solucionar as questões que afetam a saúde dessa população, dentre estas, o racismo, um dos principais determinantes sobre a saúde da população negra (BRASIL, 2011). Entender as prioridades e necessidades em saúde dessa população propicia um olhar diferenciado ao cuidado e atenção à saúde necessária que esse grupo populacional tem direito. A Saúde da População Negra não é uma área de conhecimento priorizada nos atuais cursos da área da Saúde, apesar de essencial para a formação dos profissionais de saúde e para a própria população negra (WERNECK, 2016).

A produção de conhecimento científico nessa área é inexpressível, além de serem insuficientes os dados (demográficos, econômicos, sociais) para produzir referencial teórico sobre o tema (WERNECK, 2016). Um assunto imprescindível na formação de futuros profissionais da saúde merece mais atenção, tanto em nível dos docentes, que devem abordar este conteúdo em sala de aula, quanto em instâncias superiores que determinam o conteúdo programático das grades curriculares. Diante dessa escassez é possível aventar que a temática não é levada em consideração por professores e professoras, pesquisadores e pesquisadoras, estudantes e profissionais de saúde no Brasil (MONTEIRO, 2016).

Essas ausências e insuficiências podem indicar o não reconhecimento das necessidades de saúde da população negra (WERNECK, 2016) impactando em desigualdades em certos índices importantes. Um exemplo é a porcentagem de nascimentos provenientes de mães 
adolescentes de 15 a 19 anos, que foi de $29 \%$ entre os nascidos vivos negros, 1,7 vezes maior do que a de nascidos vivos brancos (BRASIL, 2005).

O mesmo estudo verificou que $62 \%$ das mães de nascidos brancos referiram ter passado por sete ou mais consultas de pré-natal em contraste com os $37 \%$ para as mães de nascidos pardos. O risco de uma criança preta ou parda morrer antes dos 5 anos por causas infecciosas e parasitárias é $60 \%$ maior do que o de uma criança branca. Também, o risco de morte por desnutrição apresenta diferenças alarmantes, sendo 90\% maior entre crianças pretas e pardas que entre brancas. Esses diferenciais de raça, cor e etnia prevalecem mesmo com a análise centrada na proporção de óbitos por causas externas (BRASIL, 2005).

O risco de uma pessoa negra morrer por causa externa é $56 \%$ maior que o de uma pessoa branca; no caso de um homem negro, o risco é $70 \%$ maior que o de um homem branco. No geral, o risco de morte por homicídios foi maior nas populações negra e parda, independentemente do sexo. A análise dos dados desse estudo permitiu constatar que as mulheres negras grávidas morrem mais de causas maternas que as brancas, a exemplo da hipertensão gestacional; as crianças negras morrem mais por doenças infecciosas e desnutrição; e, nas faixas etárias mais jovens, os negros morrem mais que os brancos (BRASIL, 2005).

E talvez, no que seja mais grave, a negligência desses estudos torna mais difícil uma tomada de atitude das instâncias governamentais, como por exemplo, a criação de programas que solucionam as necessidades em saúde para esse grupo populacional. A Pesquisa Nacional por Amostra de Domicílios (Pnad) de 2013, divulgada pelo Instituto Brasileiro de Geografia e Estatística (IBGE), aponta que o contingente populacional negro é de 52,9\% da população brasileira e o Instituto de Pesquisa Econômica Aplicada (IPEA) mostra que cerca de $70 \%$ do público de usuários do Sistema Único de Saúde (SUS) são negros (BRASIL, 2014). Diante desses dados, é evidente a necessidade de estratégias políticas para a efetivação da equidade no que tange a População Negra, que, até hoje, sofre com discriminações raciais. É valido ressaltar que essas estratégias não são apenas no atendimento e tratamento das pessoas negras, mas o incentivo à produção de conhecimento sobre essa temática.

O SUS desde a sua criação tem, dentre as suas estratégias, a Promoção da Saúde, que pretende, por meio da Política de Promoção à Saúde, promover a qualidade de vida e reduzir vulnerabilidade e riscos à saúde relacionados aos seus determinantes e condicionantes. Sendo assim, esta política não foca apenas na doença do indivíduo, mas no modo de viver, condições de trabalho, habitação, ambiente, educação, lazer, cultura, acesso a bens e serviços essenciais de cada pessoa, atendendo, assim, as necessidades sociais em saúde da população. Essa política tem por propósito abranger e atingir toda a população brasileira, mas sabemos que cada grupo 
populacional tem suas particularidades e necessidades diferentes, como é o caso da População Negra, que ainda sofre com discriminações raciais, inclusive no âmbito da saúde. Por isso, a Política de Promoção da Saúde tem como modo de pensar e de operar articulado às demais políticas e tecnologias desenvolvidas no sistema de saúde brasileiro, contribuindo na construção de ações que possibilitam efetivar a equidade em Saúde (BRASIL, 2010).

Pensando nisso, foi criada a Política Nacional de Saúde Integral da População Negra (PNSIPN) aprovada em 2006 pelo Conselho Nacional de Saúde, que tem como objetivo promover a saúde integral da população negra, priorizando a redução das desigualdades étnicoraciais, o combate ao racismo e à discriminação nas instituições e serviços do SUS. Ela também inclui ações de cuidado, atenção, promoção à saúde e prevenção de doenças, bem como de gestão participativa, participação popular e controle social. Para além disso, incentiva a produção de conhecimento, formação e educação permanente para trabalhadores de saúde, visando à promoção da equidade em saúde da população negra (BRASIL, 2013).

Dentre as diretrizes da PNSIPN, está o incentivo à produção do conhecimento científico e tecnológico em saúde da população negra, assim como fomentar a realização de estudos e pesquisas sobre racismo e saúde da população negra. Diante disso, sabemos que o incentivo está previsto politicamente, porém nem sempre é efetivado. Principalmente durante a graduação, em que a própria carência de estudo dificulta a penetrabilidade do conhecimento nas salas de aulas, em debates e no próprio enfrentamento ao racismo, incapacitando estratégias mais pontuais para a efetivação da equidade. E, para além disso, desestimula a produção de conhecimento de pessoas interessadas no assunto, que desmunidas de dados completos, científicos e atualizados ficam limitadas na realização de uma pesquisa eficaz.

É válido ressaltar que o Estudo da Saúde da população negra engloba a Lei 10.639, de 9 de janeiro de 2003, que estabelece as diretrizes e bases da educação nacional, incluindo no currículo oficial da Rede de Ensino a obrigatoriedade da temática História e Cultura AfroBrasileira. E, mesmo com mais de 10 anos sancionada, pouco se vê na prática a efetivação dessa Lei, seja no campo educacional ou no despertar da população para a temática étnico racial como um todo (SILVA, 2013).

A Lei 10.639 tornou obrigatório o ensino da História e Cultura Afro-Brasileira e Africana nos estabelecimentos de ensino fundamental e médio (BRASIL, 2003). Como desdobramento dessa lei, as Diretrizes Curriculares Nacionais para a Educação das Relações Étnico-Raciais (DCNERER) e para o Ensino de História e Cultura Afro-Brasileira e Africana ampliaram o espectro para a Educação Superior orientando a inclusão de tal temática nos respectivos cursos de graduação (BRASIL, 2004). 
Monteiro (2016) afirma que nos cursos da área da saúde, por exemplo, pouco ou nada tem se realizado no sentido de considerar o tema em pauta como conteúdo pertinente à formação dos novos profissionais da saúde. É essencial entendermos a força e o caráter da Lei 10.639/03 não apenas como uma legislação específica orientada para a população negra em si, e sim uma alteração da Lei de Diretrizes Bases da Educação que possui abrangência nacional e deve ser implantada em todas as redes de ensino públicas se privadas brasileiras, assim como pelos conselhos e secretarias de educação e pelas universidades. (GOMES, 2011).

A Lei 10.639 estabelece a discussão dos temas étnico-raciais em toda educação brasileira. É preciso incluir essa temática na formação permanente dos profissionais de saúde em cursos como medicina, enfermagem, nutrição, fisioterapia, psicologia, odontologia e outros. Logo se antevê que a ausência do tema na formação desses alunos repercute negativamente na atuação dos profissionais e dos gestores, não capacitados para entender a importância deste aspecto na elaboração das políticas públicas e também no atendimento à População Negra.

\section{Objetivos}

Esse estudo tem como objetivo detectar nos cursos de graduação na área da Saúde da Universidade Federal de Uberlândia a existência de conteúdo sobre Saúde da População Negra, seja em disciplinas obrigatórias e optativas, nas ementas e planos de ensino, cumprindo assim, a Lei 10.639 que estabelece as diretrizes e bases da educação nacional, incluindo no currículo oficial da Rede de Ensino a obrigatoriedade da temática História e Cultura Afro-Brasileira.

Além disso, pretende alertar docentes, discentes, pesquisadores e profissionais da saúde sobre a importância do estudo e do ensino da saúde da população negra, bem como construir estratégias para a aplicabilidade efetiva da Lei 10.630 nos cursos da área da saúde na UFU.

\section{Metodologia}

Para essa pesquisa, inicialmente, foi feito um estudo bibliográfico aprofundado sobre a saúde da população negra e consequentemente sobre a Lei 10.639. Em seguida, utilizando princípios da pesquisa qualitativa (MINAYO, 2007) foi realizado um levantamento de todos os cursos da área da saúde na Universidade Federal de Uberlândia nos campi de Uberlândia, e, então, a análise de conteúdo (BARDIN, 1977) das grades curriculares, ementas e planos de ensino quanto à existência de disciplinas com conteúdo abrangendo a Saúde da População Negra. 
Os cursos analisados foram: Medicina, Enfermagem, Fisioterapia, Nutrição, Educação Física, Odontologia, Psicologia e Gestão em Saúde Ambiental. Todas as grades e ementas foram pesquisadas no site de cada faculdade. Os planos de ensino do semestre letivo 2017.2 foram solicitados às respectivas coordenações de cada curso por meio de uma carta formal enviada para os e-mails de cada coordenação e entregue pessoalmente para cada coordenador de curso.

A partir desse material de estudo, foi feita a identificação das disciplinas que continham aspectos sobre a Saúde da População Negra e, posteriormente, analisada as possíveis inserções dessa temática em componentes curriculares passíveis dessa intervenção.

\section{Resultados e Discussão}

Em pesquisa, nas grades curriculares dos cursos de Medicina, Enfermagem, Fisioterapia, Nutrição, Educação Física, Odontologia, Psicologia e Gestão em Saúde Ambiental da Universidade Federal de Uberlândia não foram encontradas disciplinas obrigatórias ou optativas que explicitassem diretamente a abordagem sobre Saúde da População Negra, bem como suas particularidades e necessidades.

Mesmo com a solicitação dos planos de ensino às coordenações de curso, obtivemos respostas positivas apenas do curso de Gestão em Saúde Ambiental e do Departamento de Saúde Coletiva do curso de Medicina que nos enviou as disciplinas de competência do seu departamento. Essa limitação de planos de ensino enviados inviabilizou uma pesquisa mais aprofundada e detalhada.

No curso de Gestão em Saúde Ambiental, foi identificada a disciplina Espaço Rural e Saúde ministrada no $3^{\circ}$ período, em que é estudado a saúde coletiva em quilombolas. Nos demais componentes curriculares não foram encontrados nenhum tópico que deixasse explícita a aplicação da discussão sobre saúde da população negra.

No curso de Medicina, nas disciplinas de Saúde Coletiva II e Saúde Coletiva VII, ministradas respectivamente no $2^{\circ}$ e $7^{\circ}$ períodos, foram encontrados tópicos importantes para a saúde da população negra, como por exemplo, o impacto dos determinantes sociais e marcadores de diferença na configuração da violência na saúde da população negra, bem como os aparatos normativo e jurídico para medidas de proteção social, assistência ao cuidado integral e sócio assistenciais a esse grupo populacional. Além disso, as disciplinas incorporam assuntos sobre a Política Nacional de Atenção Integral à População Negra como garantia de equidade a essa população. 
No curso de Nutrição, dentre as opções de disciplinas optativas que os estudantes devem cursar, existe a disciplina Políticas Públicas Setoriais oferecida pelo Instituto de Economia da UFU que tem em sua ementa o estudo de políticas públicas para a promoção da igualdade racial.

Ao considerar nossa análise dos oito cursos da área da saúde da UFU com os achados acima elencados, podemos identificar o quão precário e deficitário tem sido o estudo sobre a saúde da população negra como conteúdo mínimo na formação de futuros profissionais que atuarão na área da saúde, e como isso pode impactar diretamente no atendimento, tratamento e na qualidade do cuidado que estes irão oferecer a esse grupo populacional no exercício de suas profissões (FARIA; SILVA, 2016).

Em análises e comparações às ementas e planos de ensino, pode-se perceber a diferença na qualidade da descrição dos conteúdos programáticos para determinadas disciplinas. Enquanto algumas resumiam o semestre em uma linha, outras tinham a preocupação e o interesse em discriminar, separar e citar todos os aspectos importantes e relevantes para cada componente curricular.

Com relação à escassez de resultados, podemos refletir que isso em si demonstra algo muito significativo. $\mathrm{Na}$ carta formal enviada a todas as coordenações de cursos citados anteriormente, foram detalhados os objetivos do estudo e a importância dos planos de ensino como materiais de uma pesquisa mais fidedigna. A recusa, a desconsideração e a não colaboração dessas entidades podem indicar o quão difícil é obter dados para produção de conteúdo sobre saúde da população negra, e perceber como o racismo institucional está presente nos pequenos detalhes.

A saúde da população negra, bem como as relações étnico raciais, perpassa vários aspectos e áreas de estudo. Quando a DCNERER amplia o espectro dos estudos de História e Cultura Afro-Brasileira e Africana e seus desdobramentos para a graduação e pós-graduação, não limita ou cita os cursos que precisam abordar essa temática, pois admite-se que os cursos de graduação independentemente da área de estudo precisam estudar relações étnico raciais, principalmente levando em conta o contexto histórico que o Brasil tem com essa população (PINTO; FERREIRA, 2014).

Nos cursos da área de saúde da UFU, por exemplo, podemos refletir sobre possíveis aplicabilidades da temática. Um tema de extrema importância para entender melhor sobre saúde da população negra é o reconhecimento do racismo como um determinante no processo de saúde-doença-cuidado (BRASIL, 2011). No curso de Medicina, com a disciplina Saúde Coletiva e Método I, nos cursos de Fisioterapia e Nutrição que compartilham juntos o componente curricular Saúde Coletiva I, no curso de Enfermagem na disciplina Saúde Coletiva 
I, no curso de Psicologia na disciplina Psicologia e Políticas Públicas em Saúde Mental, no curso de Odontologia na disciplina Unidade de saúde humana 4, e no curso de Gestão em Saúde Ambiental na disciplina Epidemiologia, há em suas ementas o tópico de estudo sobre o processo de saúde-doença-cuidado. Dentro destas disciplinas, por exemplo, seria possível abordar o racismo como determinante na saúde da população negra e incluir uma discussão acerca do racismo institucional no SUS.

Nos componentes curriculares que abordam grupos vulneráveis, frequentemente é falado sobre a população negra. Algo que precisa ser discutido é que a população negra não é um grupo vulnerável per si, mas está em condições de vulnerabilidade, e, consequentemente, faz-se necessário refletir sobre os determinantes que a condiciona para ocupar essa condição, além de discutir as possibilidades de mudança de conjuntura e os cuidados de saúde necessários para com essa população (TAQUETTE; MEIRELLES, 2013).

Outra questão de grande relevância se aplica sobre os principais indicadores de saúde que incidem sobre a população negra, já elucidados anteriormente, como a taxa de mortalidade materna, a taxa de frequência às consultas de pré-natal, a taxa de mortalidade infantil, a taxa de mortes por causas externas ou por homicídios, a taxa de acometimento por doenças crônicas, taxa por doenças infectocontagiosas, entre outros. Esses dados importantes podem ser abordados tanto em disciplinas que possuem tópicos genéricos sobre os indicadores de saúde quanto em disciplinas que abordem especificamente cada um dos tópicos acima.

As disciplinas relacionadas à saúde da mulher e questões reprodutivas se apresentam como uma ótima oportunidade para a discussão sobre a forma como a sociedade trata as mulheres negras e as não negras, e como isso afeta sua saúde, e ainda como isso impacta diretamente nas taxas de mortalidade materna, e de pré-natal, e ainda refletir que esse grupo sofre além do racismo também o sexismo (KERNER, 2012), o que nos chama para um cuidado ainda mais integral e humanizado.

Em disciplinas de Pediatria/Saúde da Criança ou de Patologia, há uma abertura para se falar de doenças infecciosas e parasitárias em crianças, e pode ser interessante abordar os motivos pelos quais as crianças negras são mais acometidas por essas doenças (BRASIL, 2005), o quê elas têm de diferente das crianças não negras para serem mais susceptíveis, e também discutir quais as possíveis causas desse índice, como as condições de moradia, falta de informação, falta de acesso a saúde, dentre outras. Com isso, poderia ser estendido o debate quanto aos casos de morte por desnutrição em crianças que também é maior em crianças negras (BRASIL, 2005). 
No curso de Medicina, há um tópico no plano de ensino da disciplina Saúde Coletiva II que aborda a temática das violências, item não observado em nenhuma outra ementa de outros cursos. Esse tema é muito relevante na conjuntura atual do nosso país e se apresenta como uma oportunidade para reflexão sobre as características das vítimas de violências, seu gênero, cor, orientação sexual, e quais as consequências dessa violência na saúde das pessoas atingidas, fazendo o devido recorte de raça dentro desses dados (WAISELFISZ, 2016).

Outro tema imprescindível na formação dos profissionais de saúde é o das doenças crônicas, principalmente a hipertensão arterial e a diabetes miellitus, doenças que mais acometem a população brasileira e tem maior prevalência na população negra. Porém, o que é pouco comentado em salas de aulas é que essas doenças não estão em maior prevalência na população negra por uma questão genética, mas sim por tratamento dificultado ou/e evolução agravada, e cabe a discussão de quem ou o que tem dificultado esse tratamento (BRASIL, 2013).

Referindo-se à genética, outra disciplina também presente nos cursos de saúde, seria cabível uma reflexão acerca do que é raça, o que biologicamente e fisiologicamente define a raça de uma pessoa, e discutir se a raça de uma população tem alguma relação com suas capacidades e comportamentos.

Outra disciplina é a Ética, muito apropriada para falar sobre o respeito que devemos tratar nossos pacientes e colegas de trabalho, para expor nossos preconceitos e tentar desconstruí-los, para nos despir dos nossos prejulgamentos e aprender com as diferenças. Também há a oportunidade para discutirmos sobre não tratar de forma deficitária, ou com menos ímpeto pessoas negras e nem as negar um tratamento de qualidade.

Ao se estudar Saúde do Trabalhador, caberia uma interessante discussão sobre os diferentes tipos de trabalho, quem ocupa os cargos de salários mais altos, quem ocupa os cargos com trabalho braçal, qual a cor da população que está nos índices de desemprego, e como isso se relaciona com o passado do nosso país, com a herança do período escravocrata que herdamos, além de poder debater sobre condições apropriadas e oportunidades para a população negra, além da necessidade de ações afirmativas para aumentar a inclusão racial nas instituições (YOSHIOKA; CLARET, 2014).

Em disciplinas que envolvam Epidemiologia, é crucial falar sobre a importância do preenchimento do quesito raça/cor para os estudos epidemiológicos, para pesquisas, para identificar os riscos em saúde da população e classificar as características dessa população, e consequentemente definir melhores estratégias para o enfrentamento ao racismo principalmente dentro da saúde e os índices que assombram essa população. Sabe-se que o preenchimento 
correto do quesito raça/cor é aquele em que o próprio sujeito declara a raça/cor que ele se considera (autodeclaração) e não um terceiro que esteja preenchendo os dados de acordo com o que esse terceiro julga ser (DIAS; GIOVANETTI; SANTOS, 2009).

A Política Nacional de Saúde Integral da População Negra é pouco conhecida pelos profissionais de saúde e pelos graduandos dos cursos da área da saúde, alguns até sabem da sua existência, mas não a leram (MATOS; TOURINHOS, 2018). O ideal seria conhecer e estudar sobre essa política durante a formação desses profissionais de saúde na graduação. Nas disciplinas de vários cursos que permeiam as políticas públicas, é um espaço propício para falar sobre a Política Nacional de Saúde Integral da População Negra, pois nela está um panorama da situação da saúde da população negra e ainda aponta diretrizes para uma efetividade maior para assegurar uma saúde de qualidade e melhores condições de vida para essa população.

Nas disciplinas que abordam diversidade e plantas medicinais, como é o caso do curso de Gestão em Saúde Ambiental, há uma abertura para explanar sobre a cultura da população negra e a importância das plantas medicinais e da flora de uma forma geral para as religiões de matriz africana e afro-brasileiras, e como devemos respeitar a forma que esses religiosos utilizam dessas plantas como agentes em sua saúde (PAZ, et al. 2015).

Acima foram expostas algumas potencialidades de abordagem do tema saúde da população negra, bem como as relações étnico-raciais, cumprindo a Lei 10.639. Observou-se que existem espaços em diversas áreas para esse assunto tão necessário de ser discutido e ensinado, e como essa formação afetaria positivamente os discentes dos cursos relacionados a saúde e consequentemente a população negra que futuramente terão contato com esses profissionais.

Portanto, é importante refletir sobre as possíveis causas desses conteúdos imprescindíveis não serem abordados em sala de aula, uma vez que existem diretrizes que determinam esse conteúdo na graduação, bem como espaço para essas discussões, além da urgência que esse tema necessita.

Uma provável justificativa para esse contexto é a falta de empatia e preparo dos docentes em abordar tal assunto nas disciplinas em salas de aula (GODOY, 2017). A falta de empatia vem de um longo processo histórico de desvalorização e do silenciamento do negro, que ainda está enraizado na nossa sociedade e que vimos o quão afeta a saúde da população negra, e a falta de preparo se relaciona com a formação de tal profissional que, durante a sua graduação e especializações, pouco se viu sobre o tema e consequentemente pouco se reproduziu aos seus alunos. 
Dito isso, vemos que a renovação de saberes, e a busca contínua por conhecimento deve ser uma realidade para esses docentes, bem como a atualização de informações e dos estudos epidemiológicos, visto que a saúde coletiva precisa constantemente ser estudada e atualizada de acordo com as modificações da população. Portanto, a educação permanente de professores (MIELO, 2017) é essencial para que os dados e informações sobre a saúde da população negra sejam replicados em salas de aula de graduação.

O Ministério da Saúde oferece um curso à distância on line - Saúde da População Negra, por meio da Secretaria de Gestão do Trabalho e da Educação na Saúde (SGTES) e da Secretaria de Gestão Estratégica e Participativa (SGEP) e é oferecido pela Secretaria Executiva da UNASUS. Dentre seus objetivos constam: identificar a Política Nacional da Saúde Integral da População Negra (PNSIPN) no Sistema Único de Saúde (SUS); identificar as iniquidades referentes à saúde da população negra por meio dos dados epidemiológicos, abordando o racismo institucional em todas as suas dimensões (interpessoal e pragmática); e aplicar as estratégias da comunicação culturalmente efetiva no encontro com pacientes, famílias e comunidades negras. O curso é voltado aos profissionais de saúde que atuam na Atenção Básica, especialmente aos participantes do Programa Nacional de Valorização do Profissional da Atenção Básica (PROVAB) e Mais Médicos. Além disso, também é aberto ao público, sendo ofertado para profissionais de quaisquer áreas do conhecimento que se interessem pelo tema.

A mesma universidade onde foi feita esta pesquisa conta com o Núcleo de Estudos AfroBrasileiro (NEAB), criado em 2006 e tendo como foco o ensino, a pesquisa e a extensão na área dos estudos afro-brasileiros e das ações afirmativas em favor das populações afro-descendentes, bem como na área dos estudos da História Africana e Cultura Afro Brasileira. O NEAB-UFU, atualmente aguarda a sua regularização junto à reitoria, e trata das questões de gênero, raça e etnia referentes às modificações na Lei de diretrizes e Bases da Educação Nacional de 1996, trazidas pelas leis 10.639/03 e 11.645/08, que obrigam a educação das relações étnicoraciais e das histórias e culturas afro-brasileiras, africanas e indígenas em todos os níveis de educação públicos e privados.

Diante disso, devemos admitir, que o estudo da população negra como campo de pesquisa e formulação e ação de estratégias para esse grupo populacional se torna imprescindível pela quantidade expoente de indivíduos negros presentes na população brasileira; por ser a maioria dos usuários do SUS; por compor os piores indicadores sociais e de saúde, quando estes apresentam o recorte raça/cor; pela imprescindibilidade de efetivar os pilares da universalidade, integralidade e principalmente a equidade, que tanto vem sendo 
negligenciada, especialmente do ponto de vista da justiça social; e pela obrigação do sistema em amparar essa população em todos os aspectos necessários. (WERNRCK, 2016).

Portanto, o estudo da saúde da população negra precisa ganhar espaço nas salas de aula da graduação, na formação dos futuros profissionais de saúde e na qualificação dos que já atuam na área, para vermos os resultados no baixar dos índices que incidem sobre essa população e consequentemente uma melhoria na qualidade de vida destes.

\section{Considerações finais}

Observamos nesse estudo, a deficiência do tema saúde da População Negra nos cursos da área da saúde da UFU, porém percebemos que essas deficiências apresentam o potencial de superação sob várias formas. Passar pelo processo formativo universitário sem o contato com as particularidades do tema saúde da População Negra pode ser prejudicial para os futuros profissionais de saúde. Os espaços dentro das várias disciplinas dos cursos de graduação na área da saúde podem ser otimizados e possibilitar uma abertura de discussão e aprendizagem de tal questão.

Dito isso, visibilizar estudos sobre a saúde da População Negra, bem como da necessidade de inclusão dessa temática nos currículos de Graduação na Área da Saúde é de fundamental importância. Além dos benefícios para a própria população negra, estão colocadas as potencialidades de graduandos/as desenvolverem uma visão multicultural da saúde, assim como a habilidade de estabelecer um relacionamento profissional terapêutico, culturalmente competente, firmado em uma perspectiva anti-racista e sexista. E assim, descontruir o sistema racista que persiste na nossa sociedade e, não diferentemente, nos campos da saúde e educação.

\section{REFERÊNCIAS}

BARDIN, L. Análise de conteúdo. Lisboa: Edições 70 Ltda, 1977.

BRASIL. Ministério da Educação. Lei n. ${ }^{0}$ 10.639/2003. Brasília, DF, 2003.

BRASIL. Ministério da Educação. Diretrizes Curriculares para a Educação das Relações Étnico-Raciais e para o Ensino de História e Cultura Afro-Brasileira e Africana. Brasília, DF, 2004.

BRASIL. Ministério da Saúde. Secretaria de Vigilância em Saúde. Departamento de Análise de Situação de Saúde. Saúde Brasil: uma análise da situação de saúde no Brasil. Brasília, DF, 2005. 
BRASIL. Ministério da Saúde. Secretaria de Vigilância em Saúde. Secretaria de Atenção à Saúde. Política Nacional de Promoção da Saúde / Ministério da Saúde, Secretaria de Vigilância em Saúde, Secretaria de Atenção à Saúde. - 3. ed. - Brasília, DF, 2010.

BRASIL. Racismo como determinante Social da Saúde. Secretaria de Políticas de Promoção da Igualdade Racial - Seppir/PR. Brasília, DF, 2011. Disponível em: http://www.seppir.gov.br/central-de-conteudos/publicacoes/pub-acoes-afirmativas/racismocomo-determinante-social-de-saude-1. Acesso em: 21 maio 2018.

BRASIL. Ministério da Saúde. Secretaria de Gestão Estratégica e Participativa. Departamento de Apoio à Gestão Participativa. Política Nacional de Saúde Integral da População Negra: uma política para o SUS / Ministério da Saúde, Secretaria de Gestão Estratégica e Participativa, Departamento de Apoio à Gestão Participativa. - 2. ed. - Brasília, DF: Editora do Ministério da Saúde, 2013.

BRASIL. Ministério da Saúde. Campanha de Implementação da Política Nacional da Saúde Integral da População Negra: uma política do SUS. Brasília, DF, 2014. Disponível em: http://u.saude.gov.br/index.php/o-ministerio/principal/secretarias/1104-sgep-raiz/popnegra. Acesso em: 21 maio 2018.

DIAS, J.; GIOVANETTI, M. R.; SANTOS, N.J.S. (Org.). Perguntar não ofende: Qual é a sua cor/raça/etnia? Responder ajuda a prevenir. São Paulo, 105 p., 2009.

FARIA, M.A.; SILVA, A.J. A educação das relações étnico-raciais na formação em gestão de serviços de saúde. Revista Brasileira de Ensino Superior, 34-40, jan./mar. 2016.

GODOY, E.A. A ausências das questões raciais na formação inicial de professores e a Lei 10.639/03. Rev. educ. PUC-Camp. Campinas, 22(1):77-92, jan./abr., 2017

GOMES, N. L. (Org.). Um olhar além das fronteiras: educação e relações raciais. Belo Horizonte: Autêntica, 2007, p. 111-130.

KERNER, I. Tudo é interseccional? Sobre a relação entre racismo e sexismo. Novos estudos, CEBRAP, São Paulo, n. 93, p. 45-58, julho 2012.

MATOS, C. C.; TOURINHO, F. S. Saúde da População Negra: percepção de residentes e preceptores de Saúde da Família e Medicina de Família e Comunidade. Rev Bras Med Fam Comunidade. 2018. DOI: http://dx.doi.org/10.5712/rbmfc13(40)1712.

MIELO, M. Educação permanente na academia como estratégia de formação docente: perspectivas de docentes e preceptores. 2016. Tese (Doutorado em Enfermagem Fundamental) - Escola de Enfermagem de Ribeirão Preto, Universidade de São Paulo, Ribeirão Preto, 2017. DOI:10.11606/T.22.2017.tde-18072017-084819. Acesso em: 2019-0318.

MINAYO, M.C.S. O Desafio do conhecimento: pesquisa qualitativa em saúde. 10. ed. São Paulo: HUCITEC, 2007. 406 p. 
MONTEIRO, R. B. Educação permanente em saúde e as Diretrizes Curriculares Nacionais para Educação das relações étnico-raciais e para ensino de História e Cultura Afro-Brasileira e Africana. Saúde e Sociedade, São Paulo, v. 25, n. 3, p.524-534, 2016.

PAZ, C. E., et al. Plantas medicinais no candomblé como elemento de resistência cultural e cuidado a saúde. Revista Cubana de Plantas medicinales, Cuba, v. 20, n.1. 2015.

PINTO, M. C. C.; FERREIRA, R. F. Relações Raciais No Brasil E A Construção Da Identidade Da Pessoa Negra. Pesquisas e Praticas Psicossocias, São João Del-Rei, jul./dez., 2014.

SILVA, M.; PEREIRA, M.M. Percurso da lei 10639/03 e o ensino de história e cultura africana no Brasil: antecedentes, desdobramentos e caminhos. Em Tempo de Histórias, Brasília, n. 22, p.125-135, jun. 2013.

TAQUETTE, S. R.; MEIRELLES, Z. V. Discriminação racial e vulnerabilidades às DST/Aids: um estudo com adolescentes negras. Physis Revista de Saúde Coletiva, Rio de Janeiro, n. 23, p. 129-142, 2013.

WAISELFISZ, J. J. Mapa da Violência 2016: homicídios por arma de fogo. Rio de Janeiro, FLACSO, 2016.

WERNECK, J. Racismo institucional e saúde da população negra. Saúde e Sociedade, São Paulo, v. 25, n. 3, p.535-549, 2016.

YOSHIOKA, A.P.; CLARET, E. F. O negro no mundo do trabalho: descontentamentos e lutas. Revistas Sem Aspas, São Paulo, v.3, n.1/2, jan./dez.2014.

\section{Como referenciar este artigo}

SILVÉRIO, Ana Carolina Lino; DIAS, Nicole Geovana. Abordagem da Saúde da População Negra nos cursos da área de Saúde. Temas em Educ. e Saúde, Araraquara, v. 15, n. 1, p. 24 37, jan./jun., 2019. e-ISSN 2526-3471. ISSN 1517-7947. DOI: 10.26673/tes.v15i1.12525

Submetido em: 10/01/2019

Aprovado em: 10/03/2019 